\title{
Factores claves del ecosistema emprendedor en el estado de Sinaloa aplicando el modelo "GEM"
}

\author{
Elia Haro Armenta \\ Universidad Autónoma de Occidente \\ elia.haro@uadeo.mx
}

\section{RESUMEN}

El presente trabajo tuvo como objetivo identificar los factores claves del ecosistema emprendedor en el estado de Sinaloa. La justificación se basa en conocer las condiciones en que se encuentran los emprendedores de la entidad, alineado al modelo Monitor Global de la Actividad Emprendedora (GEM). Mediante el método analítico-deductivo, de enfoque mixto, diseño no experimental y nivel descriptivo - exploratorio. La recolección de datos se basó en la encuesta (APS) y entrevista (NES). La población estuvo constituida por emprendedores adultos de Sinaloa, muestra probabilística de 382 emprendedores y 20 expertos. Los resultados indicaron la necesidad de obtener mayores ingresos e independencia como las razones claves al decidir emprender, los adultos (34-44 años) son los que mayormente toman esa decisión, un $62.8 \%$ no temen al fracaso. El 57.4\% de las empresas se consideran establecidas con actitudes de continuar incrementando los ingresos. El ecosistema de emprendimiento es estable, aunque con bases poco firmes para lograr un crecimiento a largo plazo en los negocios, al no contar con ventajas competitivas sólidas. Se concluye que, el modelo GEM sirve para describir el ecosistema de emprendimiento en el estado de Sinaloa, para diagnosticar y analizar la situación actual, mejorar las políticas económicas y desarrollar proyectos.

Palabras claves: Ecosistema, Emprendimiento, Sinaloa, Modelo GEM. 


\title{
Key factors of the entrepreneurial ecosystem in the state of Sinaloa applying the "GEM" model
}

\begin{abstract}
The present work aimed to identify the key factors of the entrepreneurial ecosystem in the state of Sinaloa. The justification is based on knowing the conditions in which the entity's entrepreneurs are, aligned to the Global Monitor of Entrepreneurial Activity (GEM) model. Through the analytical-deductive method, with a mixed approach, nonexperimental design and a descriptive-exploratory level. Data collection was based on the survey (APS) and interview (NES). The population was made up of adult entrepreneurs from Sinaloa, a probabilistic sample of 382 entrepreneurs and 20 experts. The results indicated the need to obtain higher income and independence as the key reasons for deciding to undertake, adults (34-44 years) are the ones who mostly make that decision, $62.8 \%$ are not afraid of failure. $57.4 \%$ of companies consider themselves established with attitudes of continuing to increase revenues. The entrepreneurship ecosystem is stable, although with shaky foundations for achieving long-term growth in business, as it does not have solid competitive advantages. It is concluded that the GEM model serves to describe the entrepreneurship ecosystem in the state of Sinaloa, to diagnose and analyze the current situation, improve economic policies and develop projects.
\end{abstract}

Key words: Ecosystem, Entrepreneurship, Sinaloa, GEM Model.

Artículo recibido: 02. mayo. 2020 Aceptado para publicación: 27. junio. 2020 Correspondencia: elia.haro@uadeo.mx Conflictos de Interés: Ninguna que declarar 


\section{INTRODUCCIÓN}

La transformación económica y tecnológica mundial ha potenciado el interés en las nuevas oportunidades de negocio, y el número anual de nuevos negocios se ha doblado en los últimos años, esta tendencia se refleja en el mercado de capitales, donde las empresas son protagonistas en la economía de los países (Alcaraz, 2011).

En el contexto internacional se ha demostrado la influencia que tiene la tasa de actividad emprendedora en el desarrollo económico. En México, existe la Unidad de desarrollo productivo, que es la encargada de revisar, proponer, promover, diseñar, coordinar y ejecutar la política pública de apoyo a micro, pequeña y medianas empresas (Mipymes).

De acuerdo a la Organización para la Cooperación y el Desarrollo Económicos (OCDE, 2017), los datos del Banco Mundial demuestran que México ha incrementado el número de emprendimiento considerablemente en los últimos años, los motivos por lo cual dicho crecimiento fuera posible, se debió a la disminución de los trámites para la creación de negocios y el uso de los sistemas digitales como parte de la simplificación administrativa establecida por el gobierno. Sin embargo, a pesar del incremento en el número de empresas que inician cada año, según datos del Instituto Nacional del Emprendedor (INADEM), un gran porcentaje de éstos fracasan dentro de los cinco primeros años, debido a diversas dificultades, como la falta de objetivos y planeación de los emprendedores, apoyos financieros y la baja productividad (López, 2016).

Los estudios realizados por Monitor Global de la Actividad Emprendedora (conocido como GEM por sus siglas en inglés Global Entrepreneurships Monitor) el GEM, comprenden un extenso estudio sobre el emprendimiento a nivel mundial, ofreciendo información valiosa sobre la situación emprendedora de los países. En la actualidad el emprendimiento es una de las características que determina el crecimiento, la transformación y el desarrollo de nuevos sectores económicos de una región o un país, siendo el ser humano el principal pilar (Alcaraz, 2011).

Aunque el concepto de emprendimiento no ha logrado unanimidad en la comunidad de expertos y académicos, pero en general se asocia con la creación de algo nuevo y de manera específica, con la creación de nuevas empresas, pero también se relaciona con la 
capacidad de detectar oportunidades de negocio, acceder a estas y explotarlas (Schumpeter, 1968).

El Instituto Nacional del Emprendedor (INADEM) define a los emprendedores como las mujeres y los hombres en proceso de crear, desarrollar o consolidar una micro, pequeña o mediana empresa. Por otro lado, las características de los emprendedores ofrecen comportamientos diferentes; ya que, de acuerdo con la edad de los empresarios, los jóvenes muestran características de fortaleza y de apertura a la toma de decisiones, mientras que las personas adultas suelen ser más cautelosos y conservadores (Malinowski \& Max, 2011).

Un emprendedor disfruta la acción de crear nuevas cosas al elevar su ingenio al máximo, manteniendo el firme deseo de mejorar su entorno mediante el desarrollo de acciones emprendedoras (Hernández \& Lagunes, 2015).

En México, como en muchos otros países, es necesario que los negocios se conviertan eficientes e innovadores generadores de bienes y servicios, transformadores de materia prima, innovadora y generadores de riqueza que satisfagan necesidades, responsables del entorno social y ecológico de las regiones donde se encuentran instaladas. El logro de estos propósitos se obtiene con la creación de nuevos negocios y el fortalecimiento de las empresas instaladas. Según el INEGI en el año 2015 existían aproximadamente 4 millones de empresas: 3.9 millones de tamaño micro, 79 mil pequeñas, 16 mil medianas y casi 11 mil empresas grandes.

Para saber por qué los emprendedores no logran alcanzar altos niveles de innovación que se reflejen en los tipos de empresas que crean, es necesario analizar el fenómeno de emprendimiento, tanto desde la perspectiva del individuo como desde las variables del entorno que afectan dicha actividad (Gonzalez, 2011).

Existen diversas teorías que explican el emprendimiento desde las características del individuo, visto desde la perspectiva de teorías etológicas y psicológicas las cuales sugieren que el ser humano no es independiente a las características de su entorno, sino que también posee características internas y habilidades (competencias) que le permiten enfrentarse a nuevos retos e ideas empresariales (González \& Ganaza, 2016). 
Por otro lado, el entorno o ecosistema ha sido tema de estudio para investigadores y organismos nacionales e internacionales, quienes han analizado el problema del emprendimiento en América Latina, en países particularmente similares a los de México; uno de estos exponentes es el proyecto GEM, el cual se ha consolidado por más de dos décadas como el esfuerzo más serio y de mayor cobertura a nivel mundial en la medición de la actividad emprendedora en el mundo, más de 100 países y más de 500 investigadores de todo el mundo integran la red de investigación más grande sobre emprendimiento.

El propósito del modelo GEM es estudiar el emprendimiento desde una perspectiva integral que incluye, además de las estadísticas de emprendimiento (naciente, nuevo y establecido), las motivaciones, las actitudes personales, los valores sociales y el entorno económico, institucional y social en que se desarrolla (Naranjo, Campos, \& López, 2016).

Aunque existen indicios de que se han realizado algunos estudios que definen el emprendimiento por entidad federativa, en el estado de Sinaloa no existía hasta hoy ningún estudio que midiera el contexto del ecosistema emprendedor de manera profunda, como lo establecen los indicadores del modelo GEM de ahí la necesidad de este proyecto de investigación.

En este sentido el objetivo general de la investigación es identificar los factores claves del ecosistema emprendedor en el estado de Sinaloa aplicando el modelo "GEM".

\section{METODOLOGÍA}

El método utilizado fue deductivo y analítico con enfoque mixto (cuali-cuantitativo), nivel exploratorio y descriptivo. En cuanto a la recolección de datos, se basó en la encuesta, entrevista y revisión documental. Es decir, el estudio usó la metodología empleada por GEM para medir el ecosistema emprendedor, respetándose con apego la metodología que emplean los instrumentos: la encuesta APS (Adult Population Survey) y una guía de entrevista NES (National Expert Survey), donde se aplicó a emprendedores del estado y expertos del ámbito de emprendimiento entre los periodos comprendidos entre el 2017 y 2018, respectivamente. El diseño cuestionario y la guía de entrevista utilizados en esta investigación se retomaron del estudio del GEM Regional correspondiente al año 2016 aplicado en México. 
En este estudio se analizaron dos (2) poblaciones: La población primera la conforman los emprendedores adultos de las principales ciudades del estado de Sinaloa, considerándose una muestra de 382 emprendedores, calculada por el método estadístico para poblaciones finitas y seleccionándolos a juicio cumpliendo con las condiciones de que hubieran emprendido un negocio y se encontrarán dentro del rango de edad de entre 18 a 64 años de edad, seleccionados mediante el método aleatorio proveniente de la lista proporcionada por el servidor de la base de datos del Directorio Estadístico Nacional de Unidades Económicas (DENUE) de INEGI. Asimismo, la segunda población la constituyen los expertos en el área de emprendimiento y conocedores de los diversos temas que busca analizar esta investigación, considerándose una muestra de 20 expertos. En la Tabla 1 y Tabla 2 se muestran las dimensiones e indicadores que constituyeron las guías de los instrumentos.

Siguiendo con la metodología del GEM y conforme a los instrumentos utilizados, como parte fundamental de este estudio se realiza un análisis y descripción de un total de 14 factores claves que influyen en el ecosistema de emprendimiento, siendo estos los que se buscó conocer en esta investigación.

Tabla 1. Instrumentos de recopilación de información Encuesta APS.

\begin{tabular}{|c|c|}
\hline Dimensiones & Indicadores \\
\hline $\begin{array}{l}\text { 1. El comportamiento social e } \\
\text { individual hacia el emprendimiento }\end{array}$ & $\begin{array}{l}\text { - } \text { Actitudes hacia el emprendimiento. } \\
\text { - } \quad \text { Percepciones sobre el emprendimiento. } \\
\text { - } \quad \text { Valores sociales hacia el emprendimiento. }\end{array}$ \\
\hline 2. Niveles de emprendimiento & $\begin{array}{l}\text { - Actividad emprendedora temprana y empresas establecidas } \\
\text { - Emprendimientos descontinuados }\end{array}$ \\
\hline 3. Perfil del emprendedor. & $\begin{array}{l}\text { - Razones para emprender } \\
\text { - Género / Edad / Ingreso / Nivel educativo }\end{array}$ \\
\hline $\begin{array}{l}\text { 4. Aspiraciones de crecimiento y su } \\
\text { impacto económico }\end{array}$ & $\begin{array}{l}\text { - Sector de actividad } \\
\text { - Expectativas de expansión de mercado } \\
\text { - Novedad de productos } \\
\text { - Intensidad de la competencia } \\
\text { - Uso de nuevas tecnologías } \\
\text { - Orientación internacional }\end{array}$ \\
\hline
\end{tabular}

Fuente: GEM Regional (2016) 
Tabla 2. Instrumentos de recopilación de información Entrevista NES.

\begin{tabular}{|c|c|}
\hline Dimensiones & Indicadores \\
\hline $\begin{array}{l}\text { 1.Financiamiento al } \\
\text { emprendimiento }\end{array}$ & $\begin{array}{l}\text { - Disponibilidad de capital propio } \\
\text { - Apoyo de entidades financieras }\end{array}$ \\
\hline $\begin{array}{l}\text { 2. Políticas } \\
\text { gubernamentales }\end{array}$ & $\begin{array}{l}\text { - La Política gubernamental favorece la creación de empresas. } \\
\text { - Rapidez en el trámite administrativo } \\
\text { - Los impuestos } \\
\text { - La dificultad y burocracia en tramites }\end{array}$ \\
\hline $\begin{array}{l}\text { 3.Programas } \\
\text { gubernamentales }\end{array}$ & $\begin{array}{l}\text { - La información sobre apoyos gubernamentales } \\
\text { - El número de programas gubernamentales que apoyan } \\
\text { - El comportamiento efectivo del personal de apoyo a los emprendedores }\end{array}$ \\
\hline $\begin{array}{l}\text { 4. Educación y } \\
\text { formación } \\
\text { emprendedora }\end{array}$ & $\begin{array}{l}\text { - En la enseñanza escolar se estimula la creatividad. } \\
\text { - Los sistemas de vocación profesional proporcionan información adecuada. } \\
\text { - Las universidades proporcionan información de calidad sobre } \\
\text { emprendimiento }\end{array}$ \\
\hline $\begin{array}{l}\text { 5. Transferencia de I } \\
+\mathrm{D}\end{array}$ & $\begin{array}{l}\text { - La transferencia eficiente en las universidades sobre ciencia y tecnología } \\
\text { - Existencia de apoyos científicos y para ingenieros. } \\
\text { - La existencia de subsidios y apoyos financieros }\end{array}$ \\
\hline $\begin{array}{l}\text { 6. Infraestructura } \\
\text { comercial y } \\
\text { profesional }\end{array}$ & $\begin{array}{l}\text { - Existencia de proveedores, consultores y contratistas } \\
\text { - El costo de subcontratistas } \\
\text { - Acceso al asesoramiento fiscal y legal }\end{array}$ \\
\hline $\begin{array}{l}\text { 7. Apertura del } \\
\text { mercado interno }\end{array}$ & $\begin{array}{l}\text { - Los cambios en los mercados de bienes de consumo y de bienes de } \\
\text { servicios. } \\
\text { - El acceso a nuevos mercados } \\
\text { - Los costos } \\
\text { - La legislación }\end{array}$ \\
\hline $\begin{array}{l}\text { 8. Infraestructura } \\
\text { física y de servicios }\end{array}$ & $\begin{array}{l}\text { - El acceso a los sistemas de comunicación } \\
\text { - El costo de los servicios básicos } \\
\text { - El acceso a los servicios básicos }\end{array}$ \\
\hline $\begin{array}{l}\text { 9. Normas sociales y } \\
\text { culturales }\end{array}$ & $\begin{array}{l}\text { - La valoración del esfuerzo personal } \\
\text { - El énfasis en la autosuficiencia y autonomía } \\
\text { - La estimulación al riesgo personal }\end{array}$ \\
\hline $\begin{array}{l}\text { 10. Emprendimiento } \\
\text { social }\end{array}$ & $\begin{array}{l}\text { - La situación de pobreza } \\
\text { - La influencia de los grupos y asociaciones en contra de las regulaciones } \\
\text { - La presión de los consumidores a las empresas a las necesidades sociales y } \\
\text { ambientales }\end{array}$ \\
\hline
\end{tabular}

Fuente: GEM Regional (2016)

\section{RESULTADOS Y DISCUSIÓN}

A continuación, se presentan los resultados más relevantes que se obtuvo a partir de esta investigación con base en el Modelo GEM, mismos que proporcionan una visión más clara de la situación del ecosistema de emprendimiento en el estado de Sinaloa, subrayando que las características del proceso emprendedor se muestran de acuerdo con cada uno de los factores claves de emprendimiento de este modelo.

En cuanto al comportamiento social e individual hacia el emprendimiento, tal como se muestra en la siguiente tabla, el $87.3 \%$ de las personas emprendedoras que pertenecen al 
estado de Sinaloa se auto perciben con la capacidad de iniciar un negocio, el $53.6 \%$ de las personas que se deciden a iniciar un negocio tienden a ser de hombres y el $46.4 \%$ son mujeres, donde el sinaloense se auto percibe fuerte respecto al valor que se necesita para emprender el resultado del $62.8 \%$ indicaron que no le temen al fracaso. A pesar de que se consideran valientes y seguros de sí mismos, no son muy buenos en enfrentar situaciones de perdida, los fracasos impactan en su confianza por lo cual requieren un tiempo para volver a intentar emprender un nuevo negocio.

\begin{tabular}{|c|c|c|c|}
\hline \multirow[t]{2}{*}{ Género } & & $53.6 \%$ & $46.4 \%$ \\
\hline & & Hombre & Mujer \\
\hline \multirow{2}{*}{ Percepciones sobre el emprendimiento. } & & $62.8 \%$ & \multirow{2}{*}{$\begin{array}{c}\mathbf{3 7 . 2 \%} \\
\text { Moderada Percepción }\end{array}$} \\
\hline & & Fuerte Percepción & \\
\hline \multirow{2}{*}{ Actitudes hacia el emprendimiento } & $12.7 \%$ & & $87.3 \%$ \\
\hline & & Temor para emprender & Capacidad para emprender \\
\hline
\end{tabular}

Gráfico 1. Comportamiento social e individual hacia el emprendimiento. Fuente: Elaboración propia.

En el Gráfico 2, se presentan los resultados para la dimensión Niveles de emprendimiento, donde las empresas que existen en el territorio sinaloense, según su fase de desarrollo, el $57.4 \%$ se consideran como ya totalmente establecidas. Por otro lado, el $65.7 \%$ de la población emprendedora no ha tenido la necesidad que cerrar negocio o descontinuar algún emprendimiento lo cual sugiere que una vez iniciado proyecto es probable mantenerlo.

\begin{tabular}{|lcc|}
\hline Enpresas establecidas en Sinaloa & $57.4 \%$ & $\mathbf{4 2 . 6 \%}$ \\
& Enpresas establecidas & No establecidas \\
Necesidad de cerrar el negocio & $65.7 \%$ & $\mathbf{3 4 . 3 \%}$ \\
& NO & SI \\
\hline
\end{tabular}

Gráfico 2. Niveles de emprendimiento.

Fuente: Elaboración propia.

En el Gráfico 3, se muestran los resultados para la dimensión Perfil del emprendedor, donde las razones que impulsa al sinaloense a emprender negocio son por la necesidad de incrementar los ingresos y obtener independencia (50.7\% de los emprendedores) los ingresos que perciben no son los suficientes para mantener el estilo de vida deseado, lo cual tiene relación con el nivel de ingreso, ya que el 58.8\% de los encuestados están situados en el nivel medio. Mientras que, el $89 \%$ de la población emprendedora tiene 
nivel de educación desde la preparatoria hasta la universidad. Solamente un $6.8 \%$ de la población cuenta con conocimientos del nivel básico.

\begin{tabular}{|c|c|c|c|c|c|c|}
\hline \multirow{4}{*}{$\begin{array}{l}\text { Razones para emprender } \\
\text { Edad para emprender un negocio }\end{array}$} & \multicolumn{2}{|r|}{$50.7 \%$} & \multicolumn{4}{|c|}{$49.3 \%$} \\
\hline & \multicolumn{2}{|c|}{ Incrementar ingresos } & \multicolumn{4}{|c|}{ Mantener estilo de vida } \\
\hline & \multirow{2}{*}{$\begin{array}{l}\mathbf{9 . 7 \%} \\
18-24 \text { Años }\end{array}$} & $32.6 \%$ & \multicolumn{2}{|c|}{$34.5 \%$} & $15.6 \%$ & $7.5 \%$ \\
\hline & & 25-34 Años & -35-44 Años & 45-54 Años & \multicolumn{2}{|c|}{ - 55-64 Años } \\
\hline
\end{tabular}

Gráfico 3. Perfil del emprendedor.

Fuente: Elaboración propia.

La dimensión Aspiraciones de crecimiento y su impacto económico, las empresas consideradas como "establecidas" se dedican mayormente a ofrecer sus productos bienes y servicios a otras empresas y no a los consumidores finales, los cuales carecen de dominio de tecnología; tal como se demuestra en el Gráfico 4, cuyos resultados la actividad emprendedora temprana tiene mayor participación en el sector de servicios orientados a consumidores (43.4\%), mientras que las empresas establecidas tienen mayor participación en el sector de servicios orientados a negocios (69.6\%).

En este sentido, los emprendedores Sinaloenses, el deseo de expansión de mercado se traduce en el posible incremento de ventas, para lo cual aspiran a ello tanto la actividad emprendedora temprana como las empresas establecidas. Por otro lado, el $81.1 \%$ la actividad emprendedora temprana afirmó que sus productos ofrecidos son novedosos.

\begin{tabular}{|c|c|c|}
\hline Sector de actividad: Emprendera Temprana & $43.4 \%$ & $56.6 \%$ \\
\hline Sector de actividad: Emprendera Temprana & $\begin{array}{r}69.6 \% \\
\text { Negocios }\end{array}$ & Otros $\quad \mathbf{3 0 . 4 \%}$ \\
\hline $\begin{array}{l}\text { Expectativas de expansión de mercado (Actividad Emprendedora } \\
\text { Temprana) }\end{array}$ & $77.2 \%$ & $22.8 \%$ \\
\hline $\begin{array}{l}\text { Expectativas de expansión de mercado (Actividad Empresas } \\
\text { Establecidas) }\end{array}$ & $\begin{array}{l}65.5 \% \\
\text { Expectativas Altas }\end{array}$ & $\begin{array}{r}34.5 \% \\
\text { Expectativas Baias }\end{array}$ \\
\hline Novedad de productos (Actividad Emprendedora Temprana) & $\begin{array}{l}\mathbf{8 1 . 1 \%} \\
\text { Expectativas Altas }\end{array}$ & Expectativas Bajas $18.9 \%$ \\
\hline Novedad de productos (Actividad Empresas Establecidas) & $\begin{array}{r}\mathbf{7 5 . 4 \%} \\
\text { Expectativas Altas }\end{array}$ & Expectativas Bajas \\
\hline $\begin{array}{l}\text { Intensidad de la competencia (Actividad Emprendedora } \\
\text { Temprana) }\end{array}$ & $\begin{array}{c}64.5 \% \\
\text { Expectativas Altas }\end{array}$ & $\begin{array}{r}35.5 \% \\
\text { Expectativas Baias }\end{array}$ \\
\hline Intensidad de la competencia (Actividad Empresas Establecidas) & $\begin{array}{c}69.4 \% \\
\text { Expectativas Altas }\end{array}$ & $\begin{array}{l}\mathbf{3 0 . 6 \%} \\
\text { Expectativas Bajas }\end{array}$ \\
\hline $\begin{array}{l}\text { Intensidad de la competencia (Actividad Emprendedora } \\
\text { Temprana) }\end{array}$ & $\begin{array}{l}\text { 34.5\% } \\
\text { Exnectativas Altas }\end{array}$ & $\begin{array}{l}65.5 \% \\
\text { Expectativas Baias }\end{array}$ \\
\hline Uso de nuevas tecnologías (Actividad Emprendedora Temprana) & $\begin{array}{l}85.9 \% \\
\text { Expectativas Altas }\end{array}$ & $\begin{array}{l}14.1 \% \\
\text { Expectativas Baias }\end{array}$ \\
\hline Uso de nuevas tecnologías (Actividad Empresas Establecidas) & $\begin{array}{c}68.0 \% \\
\text { Expectativas Altas }\end{array}$ & $\begin{aligned} & 32.0 \% \\
& \text { Expectativas Baias }\end{aligned}$ \\
\hline
\end{tabular}

Gráfico 4. Aspiraciones de crecimiento y su impacto económico. 
Fuente: Elaboración propia.

Para dar respuesta al segundo objetivo específico, los resultados indicaron que la mayoría de los emprendedores que acuden a las incubadoras carecen del capital propio para iniciar su negocio, o éste es mínimo. En este sentido, hay evidencia de que en Sinaloa si se cuenta con una amplia variedad de programas de financiamiento que promueve y otorga el gobierno a través de instituciones como INADEM (Instituto Nacional del Emprendedor), NAFIN (Nacional Financiera), FND (Financiera Nacional de Desarrollo), entre otros. Por otro lado, las cuatro ciudades donde se hizo la investigación de campo se pudieron constatar que actualmente se cuenta con profesionales para asesorar a los negocios en aspectos legales, contables, fiscales, y publicitarios, entre otros.

En lo referente a las Políticas gubernamentales, a pesar del desarrollo positivo que ha tenido el apoyo al emprendimiento, no todas las localidades del estado se ven igualmente beneficiadas, a los emprendedores de muchas de estas ciudades se les dificulta llevar a cabo los trámites burocráticos para obtener las licencias que marca la ley para desarrollar empresas nuevas y en crecimiento, representando aún una gran dificultad, a pesar de que se ha estado buscando mejorar.

En general existe poco conocimiento por parte de los emprendedores de los programas de apoyo al emprendimiento., donde el uso incorrecto de los recursos financieros no es solo por parte de los emprendedores, sino también de algunos funcionarios públicos que utilizan la bolsa de financiamiento para fines propagandísticos o para favorecer a terceros. En este sentido, al asignar los recursos para cumplir compromisos con amigos y parientes es una injusta realidad que molesta y desanima a los verdaderos emprendedores que deseaban obtener el recurso para poder emprender.

La formación de los universitarios, carece de profesores con experiencia en emprendimiento. Que hayan llevado a la práctica un proyecto, alguna vez en su vida. Una vez terminadas las ferias de emprendimiento, en su mayoría, todo termina con una calificación y no existe un seguimiento y motivación para que los universitarios lleven a la práctica su emprendimiento. De este modo, los universitarios carentes de pasión, decisión e iniciativa para llevar a la realidad sus ideas emprendedoras. 
Aun sabiendo que nos enfrentamos a los retos de la globalización y la competitividad que exige el uso de tecnologías de la información y la innovación, un porcentaje los individuos, empresas y universidades no lo consideran prioridad. Es decir, una empresa puede incrementar las ventas y ampliar su cobertura de mercado, incluso diversificar sus estrategias comerciales si lo hace a través de redes, diseño de software o si implementa alguna tecnología. Pero el emprendedor aún no lo tiene claro. Existe mínima vinculación entre el sector educativo y el sector productivo en áreas de Transferencia I+D. Las empresas nuevas y en crecimiento tienen el mismo acceso a las nuevas investigaciones y tecnologías que las ya establecidas, sin embargo, solo las empresas grandes lo hacen.

Existe una amplia oferta de infraestructura comercial y profesional, sin embargo, la capacidad de asumir los costos de los honorarios de los consultores, proveedores y subcontratistas va a estar en función de la fase de desarrollo en que se sitúen las empresas de reciente creación cuentan con un presupuesto mayormente limitado, que se traduce en contratar el mínimo de servicios profesionales para comenzar o desarrollar su emprendimiento. Por otro lado, las empresas establecidas poseerán mayor presupuesto para poder contratar un mayor número de servicios profesionales.

El mercado interno en Sinaloa se cuenta con una amplia oferta de servicios particulares de profesionales en la iniciativa privada; sin embargo, representan un costo elevado en sus asesorías y programas de capacitación. En este rubro pudiese haber un área de oportunidad para que el gobierno del estado articule este tipo de despachos con los emprendedores subsidiando la contratación de estos servicios.

Tradicionalmente la sociedad valora y persigue el éxito, y puede ser tanto el afán de lograrlo que puede desarrollarse un sentimiento de envidia cando alguien más lo posee. Contrariamente a lo que se pudiera decir un número significativo de individuos sienten cierto grado de satisfacción cuando otro fracasa en un proyecto o enfrenta un problema, sin embargo, ninguna persona está exenta de fracasar pues el éxito se compone precisamente de muchos intentos fallidos, el éxito solo se alcanza con perseverancia y firmeza en la decisión de triunfar.

Existe una escaza participación de emprendimientos sociales en beneficio de la sociedad porque solo se cuenta con 87 empresas en el estado registradas con el distintivo de 
socialmente responsables. De este modo, no todos cuentan con los recursos económicos para operar, como es el caso de los centros de rehabilitación de adicciones, los cuales recurren a las calles para pedir dinero a las personas. Esto se debe en parte, a que no reciben financiamiento del gobierno y eso lo obliga a salir a las calles.

\section{CONCLUSIONES}

En definitiva, en conocer el ecosistema de emprendimiento ayuda a mejorar las políticas económicas y desarrollar proyectos que fomenten estas acciones en los estados y regiones, permitiendo con ello generar condiciones más beneficiosas en la economía y en la sociedad.

El haber implementado el método de estudio GEM de emprendimiento, como una propuesta metodológica para el análisis del ecosistema de emprendimiento en Sinaloa ha permitiendo contar con datos específico sobre las diferentes etapas del proceso emprendedor, se ha logrado identificar las características, motivaciones y ambiciones de los emprendedores, así como las actitudes sociales ante el emprendimiento y la calidad del ecosistema nacional en apoyo al emprendimiento.

Los resultados fueron poco concluyentes respecto a emprendimiento social y lo relacionado con emprendimientos sustentables, lo cual es conveniente considerar como áreas de oportunidad para continuar con futuras investigaciones en virtud a la necesidad de contar con sustentos teóricos confiables que faciliten la toma de decisiones a los gobiernos y público en general para decidir emprender negocios en esos rubros.

El ecosistema de emprendimiento en el estado de Sinaloa, es estable, pero con bases poco firmes para lograr un crecimiento a largo plazo de los negocios que emprenden, al no contar con ventajas competitivas solidas. Falta un mayor compromiso del gobierno para trabajar con las instituciones dedicadas a apoyar y promover el emprendimiento en el país y en el estado, así como la generación de educación emprendedora que logre profesionalizar y capacitar sobre la administración o gestión de nuevos negocios.

Por lo que se concluye que, el empleo del modelo GEM para analizar el ecosistema de emprendimiento en el estado de Sinaloa otorgo una visión, de igual manera el análisis de 
datos permitió a su vez obtener información cualitativa que muy útiles para diagnóstico y análisis de la situación actual del ecosistema en el estado de Sinaloa.

\section{BIBLIOGRAFÍA}

Alcaraz, R. (2011). El emprendedor de exito. (4ta Ed.). México D.F., Mexico: McGrawHill/Interamericana Editores, S.A. de C.V. Recuperdado de: https://www.stodomingo.ute.edu.ec/content/102001-322-1-2-2-

8/TEXTO_GUIA_1_El_e_m_p_r_e_n_d_e_d_o_r_de_E_x_i_t_o.pdf

González, F. y Ganaza, J. (2016). Fundamentos de Economía de la Empresa. (2da Ed.) Ediciones Pirámide. Recuperdado de:

https://www.edicionespiramide.es/libro.php?id=5151287

Gonzalez, M. (2011). The role of human capital in opportunity identification in Mexico: A gender perspective. Doctoral Dissertation. Monterrey, México: Tecnológico de Monterrey. Recuperdado de:

http://acacia.org.mx/busqueda/pdf/THE_ROLE_OF_A_PRIVATE_UNIERSITY _IN_THE_STATE_OF_ENTREPRENEURSHIP_IN_MEXICO.pdf

Hernández, K. \& Lagunes, L. (2015). Actitudes emprendedoras de los estudiantes universitarios. Recuperado de:

https://scholar.google.com.mx/citations?user=6RtWmHYAAAAJ\&hl=es.

Hernández, K., González, L., \& Herrera, M. (2016). GEM, Reporte Regional 2015 México. Reruperado de:

https://www.editorialdigitaltec.com/materialadicional/reporteregionalgem2015.p df

Instituto Nacional del Emprendedor (OCDE). Unidad de desarrollo productivo. Recuperado de: https://www.inadem.gob.mx/

López, A. (2016). El ADN del ecosistema emprendedor mexicano. Revista Digital TecReview. Recuperado de http://tecreview.itesm.mx/el-adn-del-ecosistemaemprendedor-mexicano/

Malinowski, S., \& Max, E. (2011). Integración del Emprendimiento en la Gestión del Conocimiento. Recuperado de:

https://books.google.com.mx/books/about/Integracion_Del_Emprendimiento_en _la_Ges.html?id=sQkyAwAAQBAJ\&redir_esc =y

Naranjo, E. , Campos, M., \& López, L. (2016). GEM Reporte nacional Mexico 2015. Recuperado de: https://www.gemconsortium.org/report/gem-mexico-2015national-report 
Factores claves del ecosistema emprendedor...

Organización para la Cooperación y el Desarrollo Económicos (OCDE, 2017). OCDE. Facilidad y rapidez en la creación de empresas en México. Recuperado de: https://www.oecd.org/centrodemexico/medios/43843217.pdf

Schumpeter, J. (1968). The Theory of Economic Development. Cambridge: Harvard University. Recuperado de:

https://books.google.com.mx/books?hl=es\&lr=\&id=2ieuCwAAQBAJ\&oi=fnd\&pg=PA $1 \& \mathrm{dq}=$ The + Theory + of + Economic + Development\&ots $=7 \mathrm{vS}--$

UIT4v\&sig=W7SgnQBmvXgSwqnpnzqWJf41nKI\#v=onepage\&q=The\%20Theory\%20 of\%20Economic\%20Development\&f=false 